\title{
Vibration technologies for producing metal powders
}

\author{
Margarita Cherkasova ${ }^{1}$, Alexandr Samukov ${ }^{2}$, Sergey Dmitriev ${ }^{3}$ \\ REC "Mekhanobr-Tekhnika", Saint Petersburg, Russia \\ ${ }^{1}$ Corresponding author

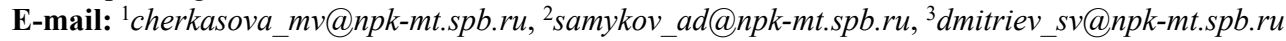

Received 29 April 2019; accepted 8 May 2019

DOI https://doi.org/10.21595/vp.2019.20757

Check for updates

Copyright (C) 2019 Margarita Cherkasova, et al. This is an open access article distributed under the Creative Commons Attribution License, which permits unrestricted use, distribution, and reproduction in any medium, provided the original work is properly cited.

\begin{abstract}
The history of application of vibratory devices for the processing of metal waste is considered. The report demonstrates the possibility of grinding metal waste generated in metalworking production on the example of iron-nickel and steel chips and of subsequent production of metal powders for additive manufacturing using the vibratory disintegration method. For the first time, the dependences of the main indicators of vibratory grinding of metal shavings on the degree of its mechanical processing were established, which made it possible to determine the specific energy consumption for the production of the required size classes. The regularities between the time spent on crushing metal shavings and power consumption during crushing are determined, which allows to detect the close relationship between energy consumption parameters and the physicomechanical properties of the chip, which makes it possible in the future to carry out a more detailed correlation of its physical and mechanical properties during disintegration.
\end{abstract}

Keywords: additive manufacturing, metal powders, metal chips, crushing, grinding, vibration effects, unbalanced vibration exciter, vibration effect, vibratory destruction mechanics.

\section{Introduction}

With the increasing demand for additive manufacturing, the market for raw materials used in the manufacture of metal powders directly applied in 3D printing is also growing. It is now possible to reuse the waste generated in metalworking of aviation and aerospace parts. The advantage of such wastes is due to the composition of the initial alloys used for manufacturing the parts: they possess the required physical properties and correspond to all technical regulations of the priority industries. High-alloyed waste may be recycled into high-grade powders, bypassing the pyrometallurgical processing, with significantly less resources, while generating several orders lower air emissions and less toxic waste as compared to the traditional processes used for obtaining such powders out of mineral raw materials [1-3].

Mechanical grinding of the feed material in vibrating or rotating ball mills is the most widely applied method for production powder. In almost all traditional devices used in the world, the load application is concentrated, and the application area and magnitude are random. In these conditions, random destruction of the crystal blocks is observed, which depends little on the defects in their structure. Moreover, ball milling itself is generally highly energy-intensive, time-consuming and inefficient [4].

This article discusses certain intermediate results of the research aimed at studying the specific features of grinding metal chips of high alloys to obtain powders with the particle size of less than $125 \mu \mathrm{m}$. In addition, certain consideration is given to the search for methods to establish physical and mechanical properties of metal chips that would enable indirect identification of the hardness, elasticity and destructibility of any metal.

\section{Initial research phase on vibratory disintegration of metal chips}

For the purpose of the research, Mekhanobr-Tekhnika REC developed a special vibratory disintegration process flow diagram that includes crushing of the initial chips to the particle size 
of less than $5 \mathrm{~mm}$ in a high-speed impact rotary disintegrator with their subsequent machining in a vibrating inertial cone crusher (KID) and further grinding in a vibratory grinding machine to the particle size of less than $0.125 \mathrm{~mm}$.

The scientific approach to the problem of the destruction of rocks as solids possessing a multiphase structure allowed Mekhanobr to develop a vibratory disintegration method based on the heterogeneous nature of materials, in particular their structural microdefects, and on the impact generated using combined vibration and pulse compression with a simultaneous shear. This method is implemented in vibratory crushing and grinding machines of various types, which include inertia cone crushers and vibrating mills [5].

Inertia cone crushers (see Fig. 1) operate as follows. Rotation of the unbalanced vibrator generates a centrifugal force, forcing the inner cone to perform gyratory motion on a spherical support and acquire an own centrifugal force that depends on the amplitude. The use of an unbalanced vibrator provides the KID with certain specific features as compared to, for example, eccentric crushers. Namely, the KID does not have rigid kinematic connections between the cones, which allows the inner cone to roll inside the outer cone at idle without a gap. At the same time, the generally accepted concept of idling is not quite typical for the KID, since the crushing force depends on the sum of the centrifugal forces for the unbalanced load and the inner cone during its motion, which, in turn, depends on the mechanics and is independent of the properties of the material being processed. In these conditions, no measures are required to ensure the stability of the inner cone on the spherical support at idle [6].

This design feature allows using KIDs for crushing and grinding materials of almost any strength, in particular, for the processing of chip waste and hard alloys.

A vibrating mill (see Fig. 2) is a horizontal cylindrical working drum consisting of several interconnected chambers filled with grinding media (balls, rods), supported by vibration shock absorbers, with double material feed. A vibration exciter transmits motion to the chamber along an elliptical, near-circular path. At the same time, the grinding media grind the material by impact and abrasion.

The division of the grinding chamber into compartments contributes to a more uniform distribution of the grinding load and material along the chamber length and to a longer grinding process due to material retention in the compartments, which ultimately leads to higher grinding fineness and improved yield of the fine product.

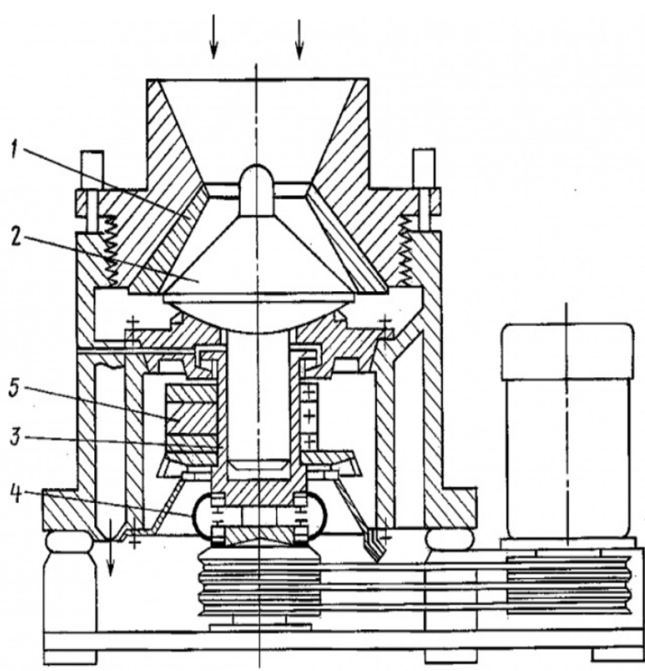

Fig. 1. KID schematic diagram:

1 - outer cone, 2 - inner cone, 3 - shaft, 4 - drive train, 5 - unbalanced vibration exciter

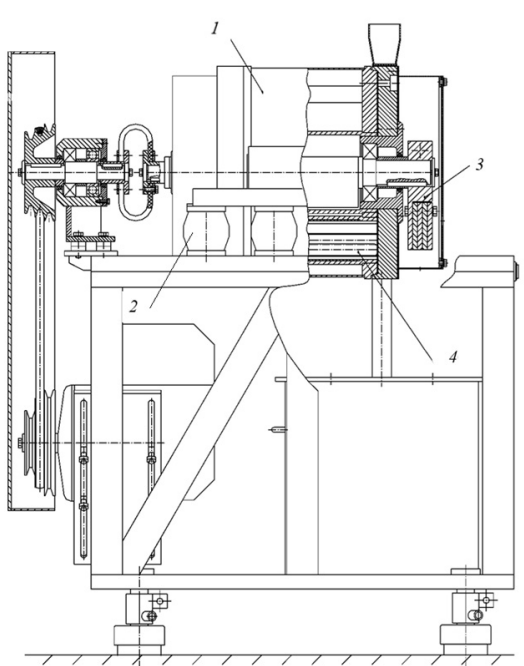

Fig. 2. Vibratory grinding machine schematic diagram: 1 - housing, 2 - vibration shock absorbers, 3 - unbalanced vibration exciter, 4 - one chamber with rollers 
In accordance with the schematic diagram, preliminary metal chips grinding studies were carried out in vibratory machines. The studies used iron-nickel alloy samples (with the nickel content of $\approx 70 \%$ ) weighing $2.5 \mathrm{~kg}$ each. A closed loop was organized for the vibratory grinding machine through discrete circulation of particles of the class of over $0.125 \mathrm{~mm}$ for four cycles without adding the feed material.

A preliminary assessment of the consequences of the impact action in the vibratory crusher for the machining degree of metal chips achieved in KID-300 and for the main grinding parameters achieved in the vibratory grinding machine demonstrated that an increase in the number of material passes through the KID leads to improved performance of the vibratory grinding machine for the classes of -0.125 and $-0.074 \mathrm{~mm}$ and reduced specific energy consumption for vibration grinding (see Fig. 3). An analysis of the results obtained also demonstrates that the specific energy consumption for the production of the $-0.074 \mathrm{~mm}$ class was two times higher than that for the production of the $-0.125 \mathrm{~mm}$ class.

A Bresser Science MTL-201 microscope was used to conduct a comparative macroscopic/optical photographic survey of the surfaces of crushed material samples.

For the material crushed in the hammer mill, the original spiral form of the chips is ground to lamellar particles with a fairly smooth surface and the minimum number of microdefects (see Fig. 4 and 6).After the vibration and pulse effects of the KID, the chip particles become thinner, down to flat particles with ragged edges, and acquire a rather contoured fibrous surface (see Fig. 5 and 7), which is apparently due to enhanced plastic shearing (strain) under the action of vibration effects and leads to work softening of the material. This reduces the strength of the metal chips, which favorably affects the energy performance of subsequent grinding.

The subsequent studies are to develop a methodology and tools for studying the vibratory destruction mechanics for KID-300 crushers and evaluating:

- The physical changes occurring in each chip when it is crushed in a KID-300 crusher, as well as in a hammer mill, using electron microscopy and X-ray microtomography;

- Specific performance of the vibratory grinding machine for the size classes of -0.125 and $-0.074 \mathrm{~mm}$, including the specific energy consumption for the production of these size classes;

- Changes in the flow characteristics and specific surface area of the crushed material.

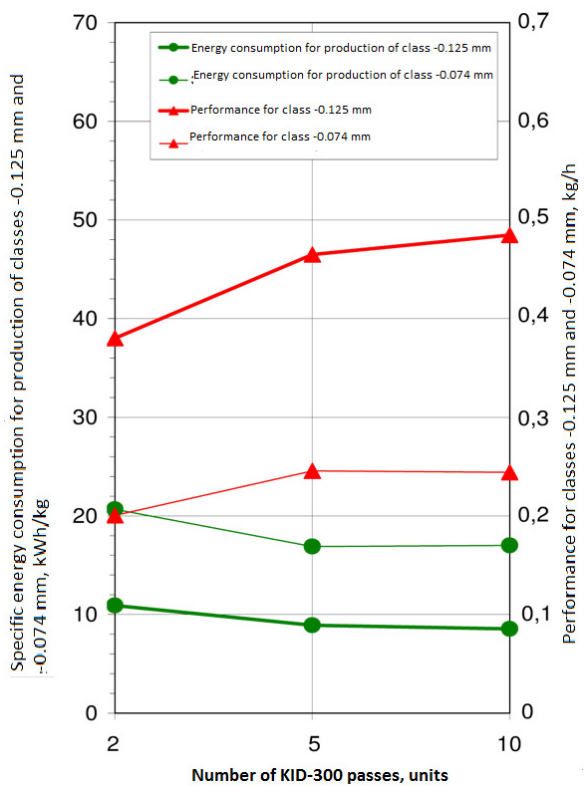

Fig. 3. Dependence of main vibration grinding indicators for metal chips achieved in a vibratory grinding machine on the degree of their machining in a KID-300 inertia cone crusher 


\section{Studies to establish physical and mechanical properties of metal chips}

The traditional methods for determining physical and mechanical properties of metals and their alloys (such as those based on hardness and tensile strength) may not be used for metal chips due to the small size and heterogeneity of structure.

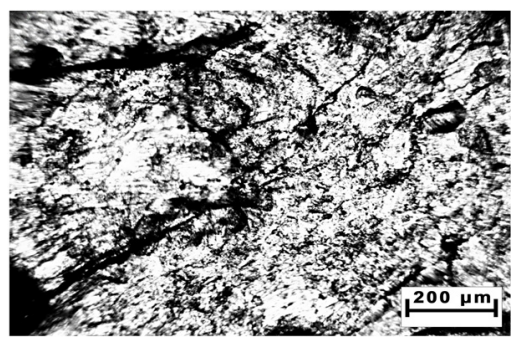

Fig. 4. Surface of metal chips after impact in a hammer mill at 10x magnification

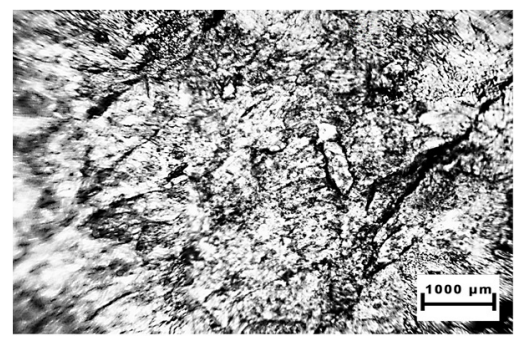

Fig. 6. Surface of metal chips after impact in a hammer mill at $20 \mathrm{x}$ magnification

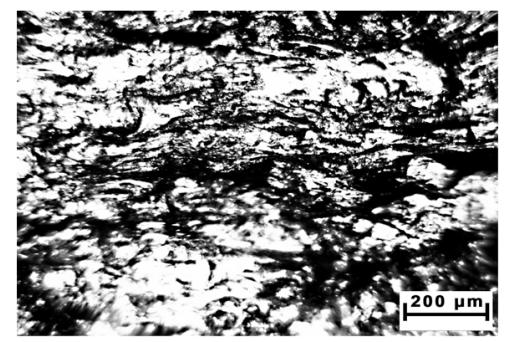

Fig. 5. Surface of metal chips after vibratory disintegration in KID at 10x magnification

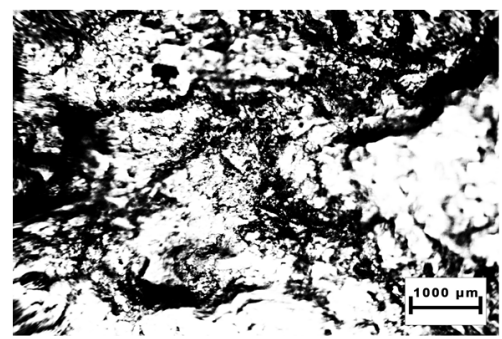

Fig. 7. Surface of metal chips after vibratory disintegration in KID at 20x magnification

The following research areas were identified as the most promising:

1) Determination of energy consumption for crushing metal chips. It is assumed that the parameters of energy consumption in the processing of metal chips will indirectly describe their hardness, elasticity and destructibility.

2) Determination of the vertical and horizontal strain in the compression of crushed metal chips and their shear in the layer. These tests will help identifying the dependence between the elasticity and hardness properties of the material and the impact and vibration effects by measuring the internal friction and viscosity of the material.

Two types of metal chips were prepared for the studies of energy consumption during metarual grinding in a high-speed impact-rotary disintegrator: iron-nickel alloy with $50 \%$ nickel content; structural carbon steel.

Two parallel samples weighing 100 grams each were selected from each specimen of prepared chips. The metal chips were crushed in a laboratory hammer crusher with the discharge mesh size of $2 \mathrm{~mm}$. The power consumption values were recorded during the crushing process (see Fig. 8). The curves for crushing different materials, as shown in Fig. 8, differ significantly, which may further allow establishing a close relationship between the energy consumption parameters when crushing metal chips and their physical and mechanical properties.

The crushed metal chips obtained earlier have sufficient particle size (less than $2 \mathrm{~mm}$ ) and structure homogeneity for their subsequent compression and shear testing, which should indirectly characterize the properties of elasticity, plasticity and hardness of the metals.

The physical and mechanical properties of the source materials were determined through evaluation of the internal friction and viscosity of crushed chips by measuring the horizontal strain (l). The measurements were conducted using a simplified method for establishing the angle of internal friction and soil adhesion according to GOST 12248-2010 using a P-10S shear device. 
The test was carried out under a continuously increasing horizontal load (kinematic regime) at a constant shear rate. The test was deemed complete if, upon application of another successive stage of the shearing load, the instantaneous shear (breakdown) of any part of the specimen with respect to its another part or the relative strain of the specimen exceeded $10 \%$.

The measurement results are shown in Figs. 9 and 10 and in Tables 1 (vertical strain) and 2 (horizontal strain).

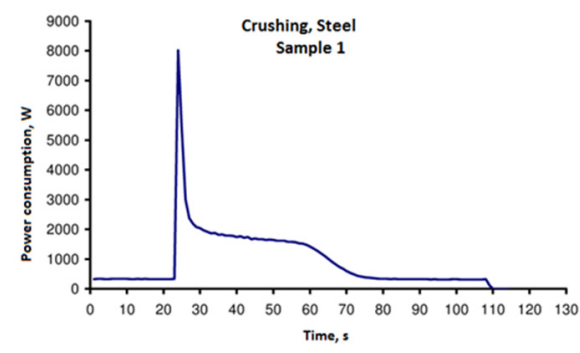

a)

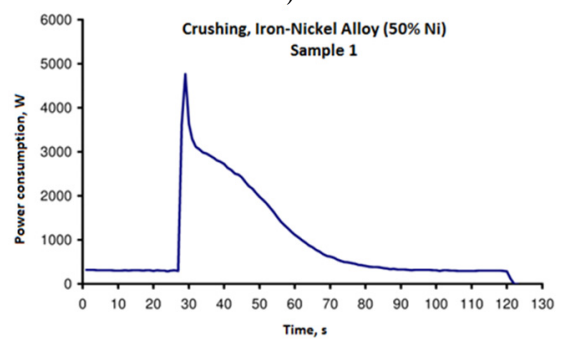

c)

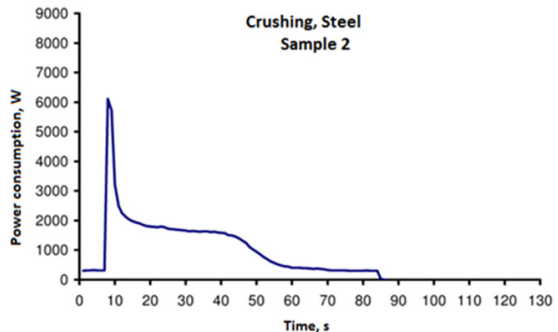

b)

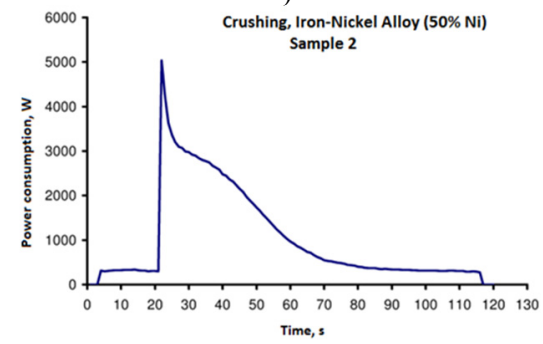

d)

Fig. 8. Diagrams of electric power consumed by hammer mill motor when crushing metal chips

The data shown in Fig. 9 suggests that the compression ratio of iron-nickel alloy chips is 1.7 times lower than that of steel chips (with the same load applied to the material), as evidenced by the change in the magnitude of the vertical strain recorded using the P-10S device.

Table 1. Vertical strain of steel and iron-nickel alloy crushed chips

\begin{tabular}{|c|c|c|}
\hline Sample & $\Delta \downarrow$ (press), $\mu \mathrm{m}$ & $\Delta \downarrow$ (press) - avg. value, $\mu \mathrm{m}$ \\
\hline Iron-nickel alloy - 1 & 22.5 & \multirow{2}{*}{22.0} \\
\hline Iron-nickel alloy -2 & 21.5 & \multirow{2}{*}{37.8} \\
\hline Steel - 1 & 37.2 & 38.4 \\
\hline Steel -2 & 38.4 \\
\hline
\end{tabular}

For the tests, two parallel samples of each material were taken.

Based on the data presented in Fig. 10, an increase in the shear stress is observed for the steel material, as evidenced by the almost twofold increase in the magnitude of the horizontal strain. At the same time, a 1.6 times higher force is required for the iron-nickel alloy strain.

Therefore, crushed metal chips made of steel have a more elastic structure under high degrees of compression, as compared to iron-nickel alloy chips.

These studies will be continued in order to develop an energy-efficient method for the destruction of high-strength materials using vibratory devices. The subsequent research are to include studies of the effects of impacts in a vibratory crusher and in an impact crusher on the grindability of metal chips in a vibratory grinding machine; the search for the best process parameters for grinding high-alloyed chips using a vibratory grinding device; and development of an industrial technology of vibratory disintegration of metal chips for the manufacture of high-grade fine powders. 


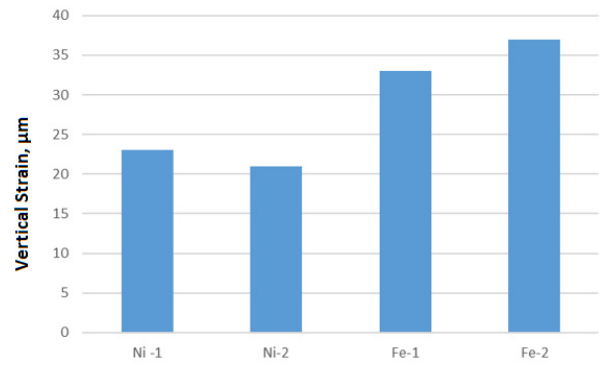

Fig. 9. Change in vertical strain

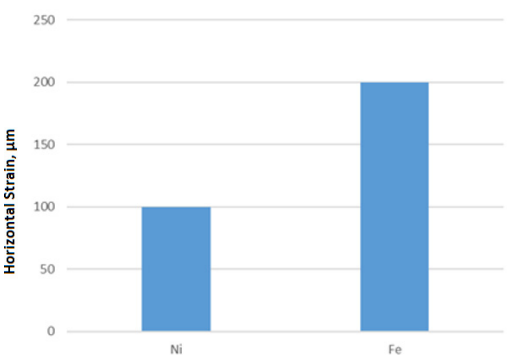

Fig. 10. Change in horizontal strain

Table 2. Horizontal strain of steel and iron-nickel alloy crushed chips

\begin{tabular}{|c|c|c|}
\hline Sample & $\Delta \leftarrow$ (shear), $\mu \mathrm{m}$ & Mass, kg \\
\hline Iron-nickel alloy & 98 & 1.0 \\
\hline Steel & 200 & 1.6 \\
\hline
\end{tabular}

\section{Conclusions}

A schematic flow diagram for vibratory disintegration was developed and preliminary studies were conducted to grind metal chips made of high alloys from their original size to the particle size less than $0.125 \mathrm{~mm}$ using vibratory crushing, grinding and screening equipment.

The use of impact vibration effects in the machining of metal chips in inertia cone crushers increases the vibratory grinding machine performance for the classes of -0.125 and $-0.074 \mathrm{~mm}$ and reduces the specific energy consumption for vibration grinding.

As part of the development and improvement of the technology for the production of powders for additive manufacturing using vibratory devices, the task has been formulated to design a methodology for establishing the physical and mechanical properties of metal chips.

In accordance with the research planned, preliminary (evaluation) studies of the physical and mechanical properties of metal chips made of iron-nickel alloy and carbon steel were carried out.

As part of the work to develop recommendations for the selection and application of vibration technologies and their industrial implementation, the prospects of their application were set with the aim of obtaining particles with the minimum particle size of up to $100 \mu \mathrm{m}$ from high alloys.

\section{Acknowledgements}

The study was carried out under the grant issued by the Russian Science Foundation (Project No. 17-79-30056).

\section{References}

[1] Dietrich S., Wunderer M., Huissel A., Zaeh M. F. A new approach for a flexible powder production for additive manufacturing. 16th Machining Innovations Conference for Aerospace Industry, 2016, p. 89-95.

[2] Bandyopadhyay A., Heer B. Additive manufacturing of multi-material structures. Materials Science and Engineering: R: Reports, Vol. 129, 2018, p. 1-16.

[3] Froes F., Boyer R. Review of additive manufacturing technologies and applications in the aerospace industry. Additive Manufacturing for the Aerospace Industry, 2019, p. 7-31.

[4] Vaisberg L. A., Safronov A. N. Vibratory disintegration application in processing of different materials. Obogashchenie Rud (Mineral Processing Journal), Vol. 1, 2018, p. 3-11.

[5] Vaisberg L. A., Zarogatsky L. P., Safronov A. N. Vibratory disintegration as the foundation of energy-saving technologies in mineral processing. Obogashchenie Rud (Mineral Processing Journal), Vol. 1, 2001, p. 5-9.

[6] Vaisberg L. A., Zarogatsky L. P., Turkin Ya V. Vibratory Crushers. Bases for Design, Engineering and Technological Applications. VSEGEI, Saint Petersburg, 2004, p. 306. 